Advances in Space Science

Vol. 1. Pp. xii +412 . (1959.) 12 dollars. Vol. 2. Pp. xiii +450 . (1960.) 13 dollars. Edited by Frederick 1. Ordway. (New York: Academic Press, Inc. ; London: Academic Press, Inc. (London), l.t.)

T

HE title of this series is misleading. It would be much more indicative of the contents if it were "Advances in Space Technology". The editor's view on 'space science' is revealed in the foreword where he states that "The principal objective of space science is to make it possible for man, and not merely instruments, one day to travel into space and to explore the planetary and satellite bodies of the solar system (and perhaps, in some undiscernible future, even the stars)".

The headings of the articles and the authors (most of whom are affiliated to American military or industrial laboratories) are as follows: Volume 1, "Interplanetary Rocket Trajectories" (D. F. Lawden), "Inter-planetary Communications" (J. R. Pierce and C. C. Cutler), "Power Supplies for Orbital and Space Vehicles" (J. H. Huth), "Manned Space Cabin Systems" (E. B. Konecci), "Radiation and Man in Space" (H. J. Schaefer), "Nutrition in Space Flight" (R. G. Tischer), "A Decimal Classification System for Astronautics" (H. H. Koelle); Volume 2, "Experimental Physics Using Space Vehicles" (C. P. Sonett), "Tracking Artificial Satellites and Space Vehicles" (K. G. Henize), "Materials in Space" (F. L. Bagby), "Plasma Propulsion Devices" (M. Camac), "Electrostatic Propulsion Systems for Space Vehicles" (E. Stuhlinger and R. N. Seitz), "Altitude Control of Satellites and Space Vehicles" (R. E. Roberson).

The articles, which are in general highly technical, are likely to be of interest mainly to professional engineers. They are not intended for, and would not be understood by, the general reader.

Author and subject indexes are supplied to each volume. The printing and production are of the customary high standard of the Academic Press.

D. R. Bates

\section{Modern Aspects of Electrochemistry}

No. 2. Edited by Dr. J. O'M. Bockris. (Modem Aspects Series of Chemistry.) Pp. ix +416 . (London: Butterworths Scientific Publications; New York: Academic Press, Inc., 1959.) 75s.

HIS book continues the survey of selected topics in electrochemistry begun in the first volume, published in 1954. There are five chapters, dealing with the present state of the theory of electrolytic solutions (by H. Falkenhagen and G. Kelbig), physical chemistry of ion exchange resins (by J. A. Kitchener), molten electrolytes (by $\mathrm{H}$. Bloom and J. O'M. Bockris), the anodic behaviour of metals (by T. P. Hoar), and the semiconductor-electrolyte interface (by M. Green). Each chapter has a full bibliography; the literature references seem mostly to end about, 1955 .

The first chapter is a very informative and detailed review of a subject which continues to attract interest. It is really a small monograph, the theory being developed from the beginning with the DebyeHückel treatment. The mathematics is often rather advanced. Activity coefficients, conduction, and (rather briefly) viscosity are dealt with, and there are many tables. The chapter on ion-exchange resins is on the lines of a small book on the subject published fairly recently. The discussion of molten electrolytes deals with a subject of increasing importance; Russian work, as well as that of the authors, find places in it. There are several tables of results, and the treatment is well balanced between theory and experiment.

The chapter on anodic behaviour of metals includes topics of technical importance, such as anodic polishing, and also deals with subjects of theoretical interest. The phenomena are still far from completely understood. The final chapter, on the semiconductorelectrolyte interface, is almost entirely theoretical, with only a brief discussion of the germanium electrode; the subject was scarcely at such a stage as to warrant a review. The book as a whole is very well written, with a good balance in each individual chapter, and good editing has brought together a most interesting selection of material.

J. R. Partington

\section{Proceedings of the International Symposium on Microchemistry}

Held at Birmingham University, August 20th to 27th, 1958. (Organized by the Midlands Section and the Microchemistry Group of the Society for Analyti. cal Chemistry.) Pp. xxvi +583. (London and New York: Pergamon Press, 1960.) 100s. net.

THIS volume consists of a collection of lectures delivered at the International Symposium of Microchemistry held at the University of Birmingharn in 1958. Many of the contributors are world-renowned in their respective fields, and the individual papers present a wealth of authoritative practical and theoretical information. Some papers also deal with the unique problems associated with the teaching of miero-analytical methods.

I almost feel it invidious to single out individual authors or topies for consideration, but the wide field covered by this remarkable book can only be illustrated in this way. F. Feigl's lecture to the whole meeting is devoted to the pyrolytic treatment of materials prior to spot-test analyses; F. J. Wolcher reviews theoretically the employment of selective organic reagents ; $\mathbf{H}$. Weisz describes the more recent 'ring oven' technique ; G. F. Hodsman and M. Corner present informative papers on 'weighing accurately' ; H. Lieb reviews forty years of quantitative organic miero-analysis, and many other experts give support. ing papers of great practical value. The practical fields of ion exchange, chromatography, polarography, radiochemistry, spectrochemistry and complexonistry are dealt with by chemists of standing, and one welcomes the papers by A. A. Benedetti-Pichler, C. J. Van Nieuwenberg and R. Beloher on the history and teaching of microchemical methods.

This is a book which will find a place not only with the individual researcher but also in the libraries of every up-to-date industrial or academic research institution. It offers, in small compass, a comprehensive survey of recent advances in widely separated fields of microchemical application and contains the valued opinions and laboratory experiences of many eminent research workers.

D. T. LEWrS 